\title{
Conflict processing in the rat brain: behavioral analysis and functional $\mu$ PET imaging using $\left[{ }^{18} \mathrm{~F}\right]$ fluorodeoxyglucose
}

\author{
Christine Marx ${ }^{1 *}$, Björn Lex ${ }^{2}$, Carsten Calaminus ${ }^{2,3}$, Wolfgang Hauber ${ }^{2}$, Heiko Backes ${ }^{1}$, Bernd Neumaier ${ }^{1}$, \\ Günter Mies ${ }^{1}$, Rudolf Graf ${ }^{1}$ and Heike Endepols ${ }^{1}$
}

\author{
${ }^{1}$ Max Planck Institute for Neurological Research, Cologne, Germany \\ 2 Department of Animal Physiology, Institute of Biology, University of Stuttgart, Stuttgart, Germany \\ ${ }^{3}$ Laboratory for Preclinical Imaging and Imaging Technology of the Werner Siemens-Foundation, Department of Preclinical Imaging and Radiopharmacy, University of \\ Tübingen, Tübingen, Germany
}

\section{Edited by:}

Jeff Dalley, University of Cambridge,

UK

\section{Reviewed by:}

Irini Skaliora, Biomedical Research Foundation of the Academy of Athens, Greece

Paul Cumming, Ludwig-Maximilians

University of Munich, Germany

Daniele Caprioli, University of

Cambridge, UK

\section{*Correspondence:}

Christine Marx, Max Planck Institute for Neurological Research, Gleueler Street 50, 50931 Cologne, Germany. e-mail: christine.marx@nf.mpg.de
Conflicts in spatial stimulus-response tasks occur when the task-relevant feature of a stimulus implies a response toward a certain location which does not match the location of stimulus presentation. This conflict leads to increased error rates and longer reaction times, which has been termed Simon effect. A model of dual route processing (automatic and intentional) of stimulus features has been proposed, predicting response conflicts if the two routes are incongruent. Although there is evidence that the prefrontal cortex, notably the anterior cingulate cortex (ACC), plays a crucial role in conflict processing, the neuronal basis of dual route architecture is still unknown. In this study, we pursue a novel approach using positron emission tomography (PET) to identify relevant brain areas in a rat model of an auditory Simon task, a neuropsychological interference task, which is commonly used to study conflict processing in humans. For combination with PET we used the metabolic tracer $\left[{ }^{18} \mathrm{~F}\right] f l u o r o d e o x y g l u c o s e$, which accumulates in metabolically active brain cells during the behavioral task. Brain areas involved in conflict processing are supposed to be activated when automatic and intentional route processing lead to different responses (dual route model). Analysis of PET data revealed specific activation patterns for different task settings applicable to the dual route model as established for response conflict processing. The rat motor cortex (M1) may be part of the automatic route or involved in its facilitation, while premotor (M2), prelimbic, and ACC seemed to be essential for inhibiting the incorrect, automatic response, indicating conflict monitoring functions. Our findings and the remarkable similarities to the pattern of activated regions reported during conflict processing in humans demonstrate that our rodent model opens novel opportunities to investigate the anatomical basis of conflict processing and dual route architecture.

Keywords: prefrontal cortex, rodent model, cognitive conflict, Simon task

\section{INTRODUCTION}

The limits of cognitive capacity are especially evident during attempts to perform simultaneously several tasks, such as speaking and typing. This leads to conflicts in the cognitive system and hence, to slower activity and in the worst case, to action slips, as typing errors. An established method to analyze such conflicts is the so called Simon task (Simon et al., 1970). For example, when a stimulus indicates that a left button press is required, a response conflict arises if the stimulus is presented on the right-hand side. The Simon effect is a neuropsychological interference effect in which reaction times (RTs) are longer and errors more frequent when spatial features of the stimulus (although task-irrelevant) are inconsistent with spatial attributes of the response (Simon et al., 1970). Contemporary models assume that the Simon effect reflects basic cognitive mechanisms in which sensorimotor processing occurs via two different channels (De Jong et al., 1994; "dual route model," Figure 1D). One route automatically activates the response spatially congruent to stimulus position ("automatic route"), whereas the other route is based on intentional selection of the response ("intentional route"). If stimulus and response position do not concur (incompatible condition), two competing responses are activated, resulting in a delayed response onset and an increased error rate (ER). While the neuronal bases of automatic and intentional pathways are unknown, human fMRI and EEG studies have suggested that the dorsal anterior cingulate cortex (ACC) monitors conflicts arising during incompatible dual route processing, and signals the dorsomedial prefrontal cortex to improve performance in subsequent conflict trials (conflict resolution; Botvinick et al., 1999, 2001, 2004; Kerns, 2006). Particularly the right inferior frontal cortex seems to participate in response inhibition as one mechanism of conflict resolution (Forstmann et al., 2008). It should be noted that there are other approaches besides the dual route hypothesis to explain the Simon effect. For example, the binding hypothesis proposes the formation of event files (Hommel et al., 2004), i.e., temporary association of cognitive representations ("codes") containing features of stimuli and response. The speed of event file formation is thought to account for variations of RTs. Another approach, the tectonic 
theory (Melara et al., 2008), suggests that inappropriate attention to the irrelevant spatial stimulus dimension disrupts selective attention to the relevant non-spatial stimulus dimension. We sought in the present study to use $\left[{ }^{18} \mathrm{~F}\right]$ fluorodeoxyglucose in order to identify the pattern of metabolic activation in the brain of rats concomitant with performance of a Simon task. As the complex manner of the Simon task requires multiple testing and imaging, an established animal model is needed. The Simon effect has already been described in rats (Courtière et al., 2007), and it was shown that metabolic behavioral positron emission tomography (PET) in animal models is a suitable method to detect activation in focal brain regions (Jang et al., 2009; Sung et al., 2009; Endepols et al., 2010). The purpose of the current study therefore was to develop an animal model that links behavior with metabolic brain activity to investigate the anatomical and functional basis of conflict processing.

\section{MATERIALS AND METHODS ANIMALS}

All animal procedures adhered to German Welfare Act and were approved by the local animal care committee and regional government authorities.

Eleven male Lister hooded rats (Harlan-Winkelmann, Borchen, Germany) were used, weighing $250 \mathrm{~g}$ at the start of the training. Animals were housed in pairs under an inverted 12:12 h lightdark cycle (lights out at $8 \mathrm{am}$ ) in a temperature- and humiditycontrolled facility room $\left(20 \pm 2{ }^{\circ} \mathrm{C}, 50-60 \%\right)$ and restricted to $15 \mathrm{~g}$ food per animal per day. Water was available ad libitum.

\section{APPARATUS FOR BEHAVIORAL TESTING}

Animals were tested in an operant chamber $(30.5 \mathrm{~cm} \times 24.1 \mathrm{~cm} \times$ $21.0 \mathrm{~cm}$; Med Associates, Inc., Georgia, VM, USA) with a central nose poke unit and two trough-like food receptacles on either side, equipped with light barriers for measuring reaction and movement times (Robbins et al., 1993). Food receptacles were connected to a motor-driven pellet dispenser, delivering 45-mg precision pellets (Bioserv) as reward. Two loudspeakers (Med Associates "cage tweeter," range: $5-15 \mathrm{kHz}$ ) were placed above the pellet receptacles (Figure 1). The acoustic stimuli consisted of two $300 \mathrm{~ms}$ (rise/fall time $5 \mathrm{~ms}$ ) pure tones with carrier frequencies of 10 and $15 \mathrm{kHz}$, and a sound pressure level of $60 \mathrm{~dB}$. All experiments took place during the animals' dark phase under red light.

\section{OPERANT CONDITIONING OF THE BASIC SIMON TASK}

On the first day of training rats were allowed to become accustomed to the operant chamber, the auditory stimuli, and the food reward (45-mg precision pellets, Bioserv). The two auditory stimuli alternated in a pseudorandomized fashion every $10 \mathrm{~s}$, and were associated with a food reward from the pellet trough on the side associated with the stimulus. From the second day on, the rats had to learn to initiate a trial by themselves with a nose poke. The nose poke should last at least $1.5 \mathrm{~s}$. Pokes under $1.5 \mathrm{~s}$ were indicated by diode illumination in the nose poke unit and punished with $2 \mathrm{~s}$ in which the rat could not start a new trial. A correct nose poke resulted in a bilateral tone presentation (i.e., one of the two stimuli was played back simultaneously from the two speakers) and immediate delivery of a pellet at the side associated with the stimulus. In the last training step, the reward was no longer delivered automatically after stimulus presentation. Instead, the rat had to choose one side according to the auditory stimulus and enter the pellet trough. Five of the eleven rats were trained to go to the left food receptacle after a $10-\mathrm{kHz}$ stimulus and to the right food receptacle after a $15-\mathrm{kHz}$ stimulus. The other six rats were trained to make the opposite association between frequency and side of reward. If the choice was correct, a pellet was delivered. If the rat chose the wrong side, the nose poke diode illuminated for $2 \mathrm{~s}$ during which the rat could not start a new trial. The procedure of this training step was identical with the control task consisting of $100 \%$ neutral trials $\left(T_{\mathrm{N}}\right)$ in behavioral PET (see below). In all other tests following the training stage, the acoustic stimulus was delivered unilaterally (i.e., one of the two stimuli was played back from one of the speakers, either left or right). Rats were trained for several weeks, always on five consecutive days and then rested for 2 days. Each training session lasted 15 min or was terminated if the rat accomplished 60 correct responses in less than $15 \mathrm{~min}$. Rats advanced to the next training step after reaching a performance level of $85 \%$ correct responses.

\section{BASIC SIMON TASK}

The basic Simon task resembled the last training step, only with unilateral stimulus presentation, and a total of 120 trials. During the basic Simon task, rats had to initialize every trial with a nose poke $>1.5 \mathrm{~s}$ in the central nose poke unit, which lead to playback of one single auditory stimulus ( $300 \mathrm{~ms}$; tone pitch 10 or $15 \mathrm{kHz}$ in pseudorandomized order). According to pitch, rats had to choose the left or right food receptacle, and were rewarded after correct choice. If the sides of stimulus presentation and correct response concurred, this was recorded as a compatible condition (C). During incompatible conditions (I), stimulus and response occurred on different sides. Only in neutral trials $(\mathrm{N})$ tone stimuli were emitted from both speakers simultaneously. Conditions were presented in a pseudorandomized sequence. The reaction time (RT; Figure 1) was taken as the time between start of the auditory stimulus and withdrawal from the nose poke unit, while movement time was taken as the time from nose withdrawal until entrance of the food receptacle. Because movement time was similar under all conditions, it will not be mentioned further in this study. The trial was terminated if the nose was withdrawn before the end of the required nose poke-time of $1.5 \mathrm{~s}$, while trials with RT $>1 \mathrm{~s}$ or $<130 \mathrm{~ms}$ were discarded off-line. Error rate (ER) was taken as the percentage of wrong choices, and was arc-sine square root transformed before statistical analysis.

\section{TEST SCHEDULE}

Two experimental blocks were conducted: one behavioral PET imaging block with five different tests, followed by one purely behavioral block with three different tests. All tests, except the resting state control, were variants of the basic Simon task (see below). Each rat had to perform all tests within a block.

\section{BEHAVIORAL PET IMAGING BLOCK}

In the behavioral PET block we wanted to see focal metabolic brain activation associated with Simon-like conflicts. Each rat underwent five behavioral PET sessions. We took the task with 


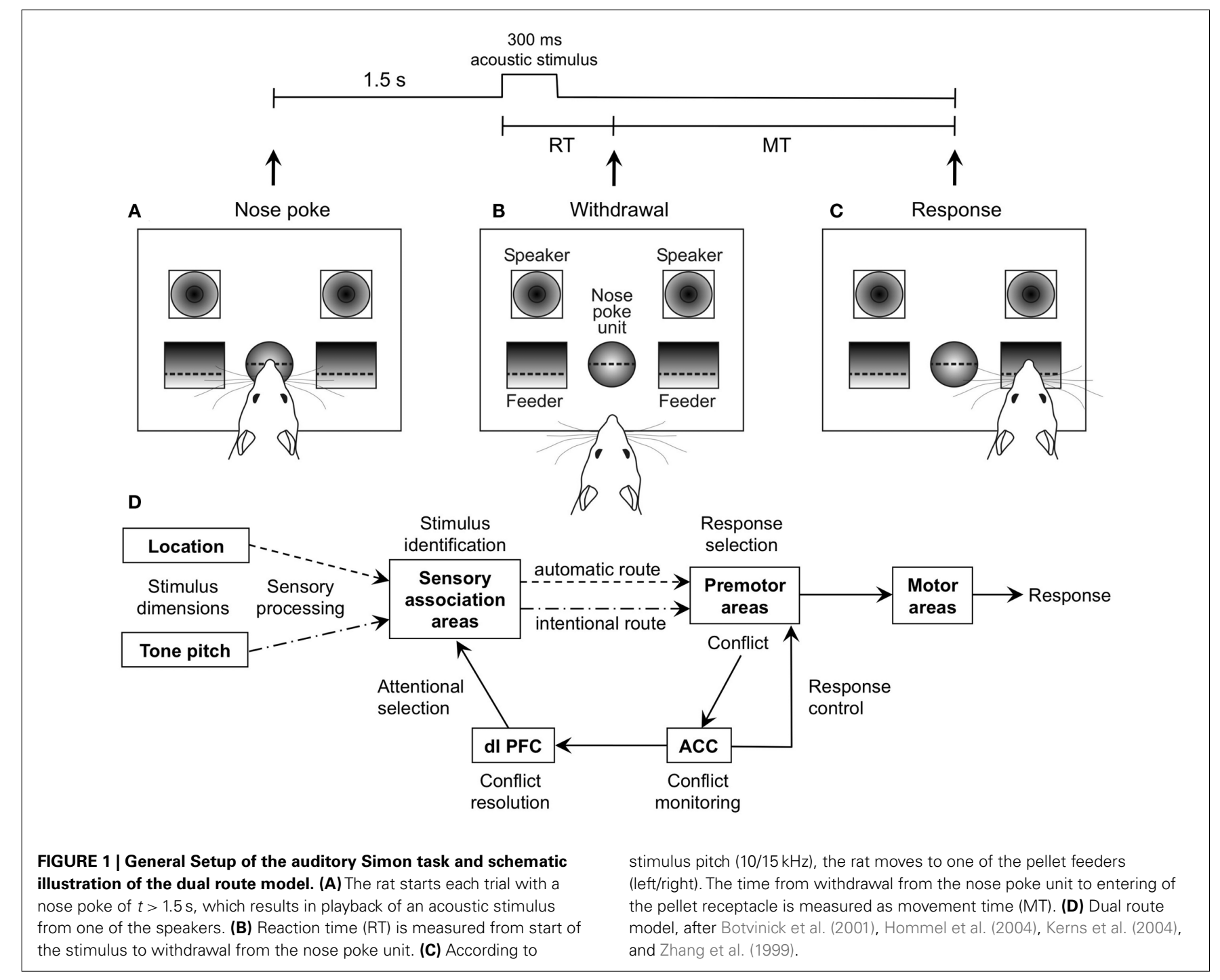

$50 \%$ compatible and $50 \%$ incompatible trials as a basis, because a balanced number of compatible and incompatible trials avoids biasing the metabolic response by one type of trials.

(1) Basic Simon task with 50\% incompatible and 50\% compatible trials in randomized order $\left(T_{\mathrm{R}}\right)$. The Simon task had to be compared to several control conditions:

(2) No-conflict control with neutral trials only $\left(T_{\mathrm{N}}\right)$. Here, the rats had to do exactly the same as during $T_{\mathrm{R}}$. However, conflicts did not occur because bilateral stimulus presentation prevented spatial information.

Because of the cumulative nature of PET it is not possible to separate metabolic responses to compatible and incompatible trials. We therefore conducted two additional controls with 100\% compatible and $100 \%$ incompatible trials, respectively:

(3) Basic Simon task with compatible trials only $\left(T_{C}\right.$; side of stimulus presentation and required response side always matched);

(4) Basic Simon task with incompatible trials only ( $T_{\mathrm{I}}$; stimulus and required response were always on opposite sides); Finally, we wanted to compare the Simon task with the naive situation before training:

(5) Resting state control $\left(R_{\mathrm{S}}\right)$, with naive rats before operant conditioning. In $R_{\mathrm{S}}$ there were no cognitive requirements, the rats only heard the sound stimuli in random order with $10 \mathrm{~s}$ interstimulus intervals and food pellets accessible ad libitum in the food receptacle.

Tasks (1)-(4) took place after successful operant conditioning. They were presented on average 6 days apart in the order of increasing complexity, i.e., $T_{\mathrm{N}}, T_{\mathrm{C}}, T_{\mathrm{I}}, T_{\mathrm{R}}$. During the days in between PET sessions, the rats repeated the last training step.

For the combination of behavior with metabolic PET imaging, rats were briefly anesthetized for intraperitoneal injections of $\left[{ }^{18} \mathrm{~F}\right]$ fluorodeoxyglucose (FDG; $1.7-2.1 \mathrm{mCi} ; 500 \mu \mathrm{l}$ injection volume; stock solution in $228 \mathrm{mM}$ Na-phosphate buffer, diluted as needed with $0.9 \% \mathrm{NaCl}$ ). Five minutes after tracer injection, rats started to perform one of the five tasks in the operant chamber for $30 \mathrm{~min}$. As a glucose analog, FDG is incorporated by active brain cells and is subsequently phosphorylated by hexokinase, but 
cannot be further metabolized because of the missing hydroxyl group (Wienhard, 2002). The process of trapping is an indicator of the state of metabolic activity of tissue, which can be measured during a scan under anesthesia after the behavioral task. Fifty minutes after FDG administration (i.e., 15 min after the end of the behavioral task), animals were anesthetized by inhalation of isoflurane ( $5 \%$, delivered in $70 \% \mathrm{~N}_{2} \mathrm{O}$ and $30 \% \mathrm{O}_{2}$ ), and placed in the animal holder of a Focus 220 micro PET scanner (CTI/Siemens Knoxville, TN, USA; resolution at center of field of view: $1.4 \mathrm{~mm}$ ). Breathing rate was kept at 50-70 per min by adjusting isoflurane concentration (1.5-2.5\%). Body temperature was held at $37^{\circ} \mathrm{C}$ with a feedback-controlled flow of warm water through the animal holder. Glucose concentration was measured in a blood sample collected from the tail vein at $60 \mathrm{~min}$ after FDG injection, using a blood glucose level meter (One Touch Ultra). Emission data were recorded over $30 \mathrm{~min}$ in list mode, starting $60 \mathrm{~min}$ after FDG injection. Following Fourier rebinning, data were reconstructed using the iterative OSEM3D/MAP procedure (Qi et al., 1998), resulting in voxel sizes of $0.38 \mathrm{~mm} \times 0.38 \mathrm{~mm} \times 0.82 \mathrm{~mm}$. RTs and ERs measured during FDG accumulation were compared over tasks (1)-(4) using one-way repeated measures ANOVA with post hoc comparison and Holm-Sidak correction. Each task provided one factor level, except task (1) $T_{\mathrm{R}}$, which yielded two factor levels, one for compatible and the other for incompatible trials.

\section{BEHAVIORAL BLOCK}

In the behavioral block we wanted to see if the Simon effect occurred in a test situation undisturbed from PET procedures, e.g., tracer injection, and if the Simon effect would be influenced by the probability of incompatible trials. All rats conducted three basic Simon tests, each with different relative probabilities of incompatible trials: (1) $20 \% \mathrm{I}$; (2) $50 \%$ I; identical to $T_{\mathrm{R}}$ in the PET block; (3) $80 \%$ I. For example, in the $80 \%$ I condition a rat trained to associate the $15 \mathrm{kHz}$ tone with the left response side and the $10 \mathrm{kHz}$ tone with the right response side heard the following in randomized order: $15 \mathrm{kHz}$ - right speaker (I) $-40 \%$ of trials; $10 \mathrm{kHz}-$ left speaker (I) $-40 \%$ of trials; $15 \mathrm{kHz}$ - left speaker (C) $-10 \%$ of trials; $10 \mathrm{kHz}-$ right speaker $(\mathrm{C})-10 \%$ of trials. A test condition with $100 \%$ incompatible trials, as used in the PET block, is not suitable for behavioral analysis, because a certain number of compatible trials is required within the same task for comparison of RT and ER.

Rats received one test session per day, in randomized order balanced between animals. Effects of conflict probability and condition on RT and ER were estimated with two-way repeated measures ANOVA (see results for factorial design) and HolmSidak corrected post hoc comparison. Statistical computations were conducted with Sigma Plot (version 11.0, Systat Software, Inc.). Significance level ( $\alpha$-level) was set at $p<0.05$.

\section{MRI SCANS}

To rule out gross structural brain anomalies and to provide individual templates for co-registration of the PET images, T2weighted structural MR images were acquired. MRI scans were performed in a 4.7-T BioSpec animal scanner (Bruker BioSpin, Ettlingen, Germany) using a quadrature transmit/receive birdcage coil (Rapid Biomedical, Rimpar, Germany) with an inner diameter of $38 \mathrm{~mm}$. A relaxation enhancement (RARE) sequence was used: RARE factor $=8, T_{\mathrm{R}} / T_{\mathrm{E}}=5000 / 14.0 \mathrm{~ms}$, averages $=2$, matrix size $=256 \times 256, \mathrm{FOV}=4.6 \mathrm{~cm} \times 4.6 \mathrm{~cm}, 21$ slices, slice thickness $=1.3 \mathrm{~mm}$, interslice interval $=1.8 \mathrm{~mm}$. Inhalation anesthesia procedures were the same as those used for $\mu$ PET scans.

\section{IMAGING DATA ANALYSIS AND STATISTICS}

MRI and PET data were analyzed using the imaging tool VINCI (Vollmar et al., 2007). MR images were manually co-registered on a master brain derived from the atlas of Swanson (2003) and examined for structural abnormalities. PET images were then manually co-registered on the corresponding MR images. With the help of the master brain, individual MR images, and the brain atlas of Paxinos and Watson (2005), three-dimensional volumes of interest (VOIs) corresponding to defined brain areas (Table 1; Figure 6) were drawn section by section in the transverse/coronal plane. Section thickness was identical with $z$-dimension of voxels, i.e., $0.815 \mathrm{~mm}$. For intensity normalization, every image was divided by the respective mean value of a whole brain VOI (ratio normalization; Arndt et al., 1996). Normalized metabolic activity was then assessed in the VOIs of individual brain areas. Because of chewing food reward pellets during operant behavior, high FDG uptake of temporal muscles was unavailable, and substantial spillover of radioactivity obscured some parts of the lateral cerebral cortex (Figure 2). With a threshold function we determined the outline of muscle activity and used this to draw a muscle artifact mask and to adjust VOIs, if necessary.

In order to assess task-relevant regional brain activation, we compared PET sessions with each other. For analyzing brain activation associated with the task condition in general, we compared
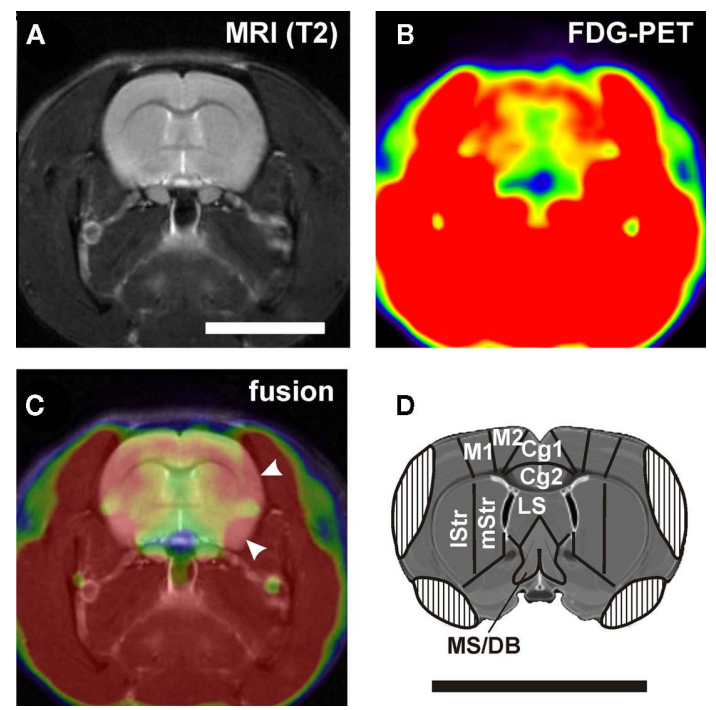

FIGURE 2 | (A) Example of a structural MRI and (B) the corresponding PET image. (C) Fusion of the two images shows muscle artifacts on the lateral aspects of the brain (arrowheads). This leads to covering of the lateral cortical regions in the atlas (D). Cg1, Cg2, anterior cingulate cortex area 1 and 2; LS, lateral septum; IStr, dorsolateral striatum; M1, motor cortex; M2, premotor cortex; MS/DB, medial septum and diagonal band of Broca; mStr, mediodorsal striatum. Scale bars: $1 \mathrm{~cm}$. 
Table 1 | Volumes of interest analysis of relative metabolic activity during the Simon task $\left(T_{\mathrm{R}}\right)$ and control tasks $\left(T_{\mathrm{C}}\right.$ and $\left.T_{\mathrm{l}}\right)$.

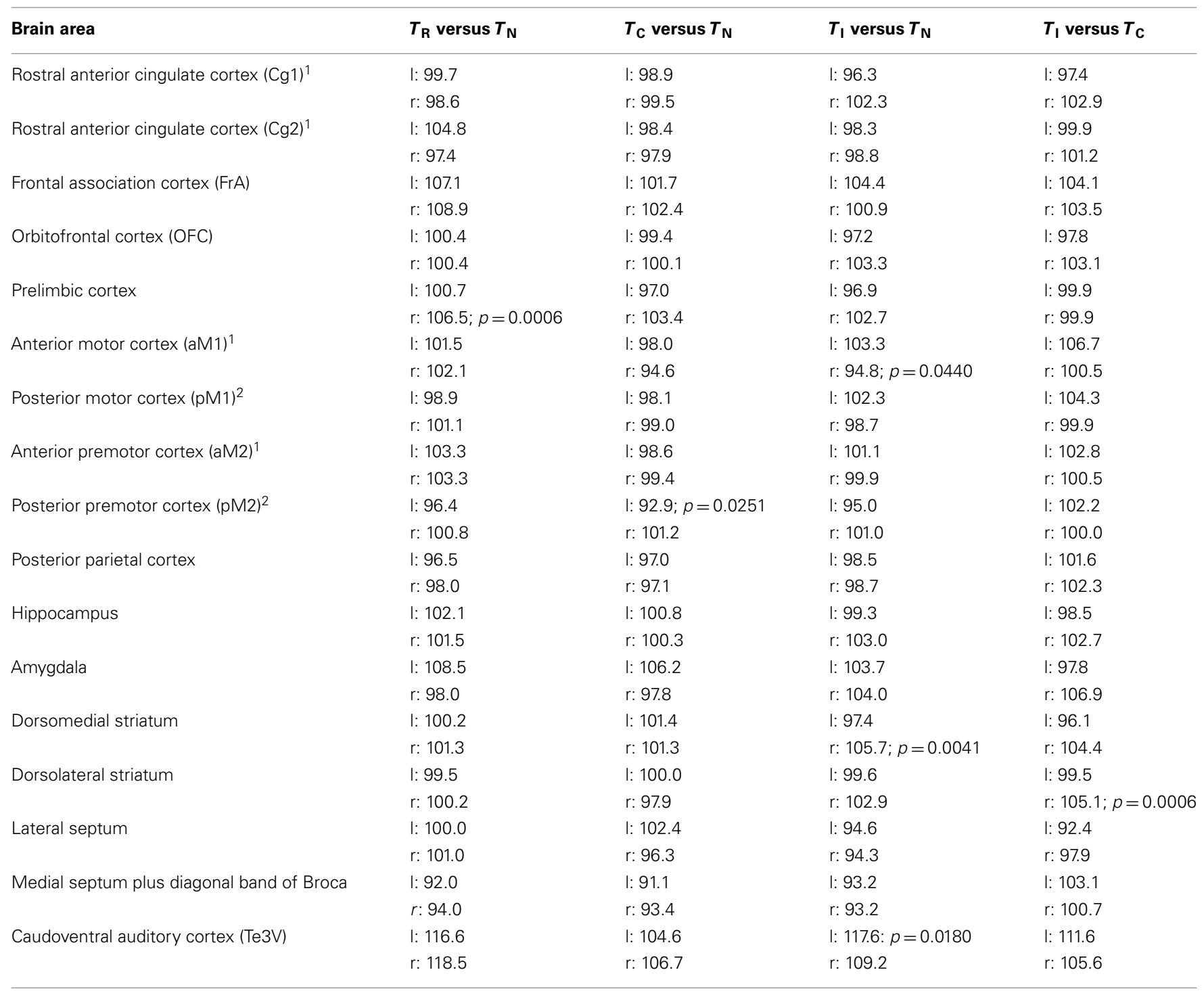

${ }^{1}$ Up to $0.6 \mathrm{~mm}$ rostral from Bregma.

${ }^{2}$ From $0.6 \mathrm{~mm}$ rostral to $2.0 \mathrm{~mm}$ caudal from Bregma.

Given values represent \% regional metabolic activity in the PET sessions $T_{R}\left(50 \%\right.$ incompatible and $50 \%$ compatible trials in randomized order), $T_{C}$ (compatible trials

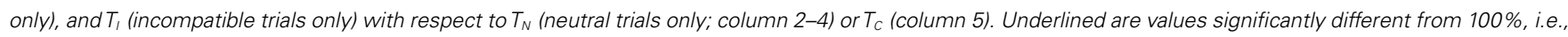
from values in $T_{N}$ (column 2-4) or $T_{C}$ (column 5). Shown are p-values uncorrected for multiple testing. I, Left hemisphere; $r$, right hemisphere.

$T_{\mathrm{I}}$ (Simon task with $100 \%$ incompatible trials) with the resting state control $R_{\mathrm{S}}$ by calculating $100 \times T_{\mathrm{I}} / R_{\mathrm{S}}$ (i.e., percent normalized metabolic activity of $T_{\mathrm{I}}$ versus $R_{\mathrm{S}}$ ). Metabolic activation associated solely with conflict processing was assessed by displaying percent metabolic activity of $T_{\mathrm{R}}, T_{\mathrm{I}}$, and $T_{\mathrm{C}}$ versus the no-conflict control $T_{\mathrm{N}}\left(100 \times T_{\mathrm{R}} / T_{\mathrm{N}} ; 100 \times T_{\mathrm{I}} / T_{\mathrm{N}} ; 100 \times T_{\mathrm{C}} / T_{\mathrm{N}}\right) . T_{\mathrm{N}}$ was chosen as reference condition, because the bilateral stimulus presentation provides ambiguous spatial information, and therefore no-conflict occurs. In $T_{\mathrm{C}}$, on the other hand, there is no-conflict, either, but it cannot be ruled out that facilitatory processes (e.g., facilitation of the automatic route) may take place. For this reason, normalized metabolic activity during the incompatible control task $T_{\mathrm{I}}$ was also compared to the compatible control task $T_{\mathrm{C}}$ as well. Using the one-sample $t$-test, we compared the resulting relative VOI activities with $\mu=100 \%$ (i.e., no change relative to $T_{\mathrm{N}}$ or $\left.T_{\mathrm{C}}\right)$. In addition to the VOI analysis, we compared matched voxels of the four tests using one-way repeated measures ANOVA. For post hoc comparison, the Holm-Sidak method was used with $T_{\mathrm{N}}$ serving as control. Voxels from $T_{\mathrm{I}}$ were also compared to $T_{\mathrm{C}}$ with the help of the paired $t$-test. Finally, correlation analyses were run between task related activity changes on the one hand, and RT and ER on the other hand, using the Pearson product moment correlation test. As the rat brain comprises approx. 19,000 voxels, voxel-based statistical calculations include multiple comparisons associated with a considerable increase in the type I error rate. $P$-values were corrected for multiple comparisons using 
the Benjamini-Hochberg control of false discovery rate. However, as in previous PET studies with low degrees of freedom (e.g., Nichols and Hayasaka, 2003; Rocke et al., 2005), all individual voxel comparisons missed significance if using the false discovery rate procedure. Uncorrected significant $p$-values were between 0.01 and 0.05 , therefore we set a threshold of $p=0.02$ [corresponding to $F(3,9)=5.51]$, as proposed by Genovese et al. (2002).

\section{RESULTS \\ TEST SCHEDULE}

Seven animals were used for behavioral PET, and successfully conducted $R_{\mathrm{S}}$ and $T_{\mathrm{I}}$. One rat died during the PET block, and two refused to work reliably in the PET situation, so that four rats completed all five PET tests. For the subsequent behavioral block, these four plus four additional animals were used.

\section{Behavioral block}

Behavioral data. We start with reporting the behavioral data, because occurrence of a stable Simon effect in the behavioral experiments undisturbed from PET procedures is the prerequisite for all further analyses. The different probabilities of incompatible trials are important for the following PET block as well, since a pronounced Simon effect with a high rate of incompatible trials would suggest a high conflict level in the PET $T_{\text {I }}$ condition (100\% incompatible trials). The analysis of the Simon tasks with three different frequencies of incompatible trials revealed a Simon effect for both [RT; $(n=8)$; mean values calculated for all three Simon tasks: $\mathrm{RT}[\mathrm{C}]=315 \mathrm{~ms}$; RT $[\mathrm{I}]=351 \mathrm{~ms}$ ] and [ER; mean values for all three Simon tasks: ER $[\mathrm{C}]=7 \%$; ER $[\mathrm{I}]=14 \%$; Figure 3]. This was confirmed by two-way repeated measures ANOVA with the factors "condition" (factor levels: I, C) and "probability of incompatible trials" (factor levels: 20\% I, 50\% I, 80\% I), indicating a significant main effect of the factor "condition" on RT $[F(1,14)=16.8$, $p=0.005]$ and $\mathrm{ER}[F(1,14)=10.5, p=0.014]$. Post hoc comparison showed that RT[C] was significantly shorter than RT[I] in the $20 \% \mathrm{I}(p=0.003)$ and $80 \%$ I task $(p=0.029)$, while ER[C] was significantly lower than ER[I] in $20 \% \mathrm{I}(p=0.001), 50 \% \mathrm{I}$ $(p=0.016)$ and $80 \%$ I task $(p=0.029)$. Conflict probability had no significant main effect on RT and ER.

\section{Behavioral PET imaging block}

Behavioral data. Here we evaluated if rats showed a Simon effect also in the PET condition, where tracer injection, scanner noise, etc., may have compromised conflict processing. Most important are results of $T_{\mathrm{R}}$, where compatible and incompatible trials can be compared directly. Four animals were tested repetitively in combination with PET in all conditions. Rats conducted 128246 trials per session. Blood glucose levels at the start of the scan (123-192 mg/dl) did not correlate significantly with trial numbers ( $R=0.17, p=0.53$, Pearson product moment correlation test), suggesting that the number of consumed food pellets during the task did not bias global cerebral FDG uptake. $T_{\mathrm{R}}$ yielded results comparable to those of the behavioral block (Figure 4). RTs were on average $33 \mathrm{~ms}$ shorter in compatible compared to incompatible trials ( $t=2.17, p=0.059$; one-tailed paired $t$-test). In the control tasks consisting of only one type of condition $\left(T_{\mathrm{C}}, T_{\mathrm{I}}\right.$, and $\left.T_{\mathrm{N}}\right)$, RTs were similar to those in incompatible trials of $T_{\mathrm{R}}$ and did

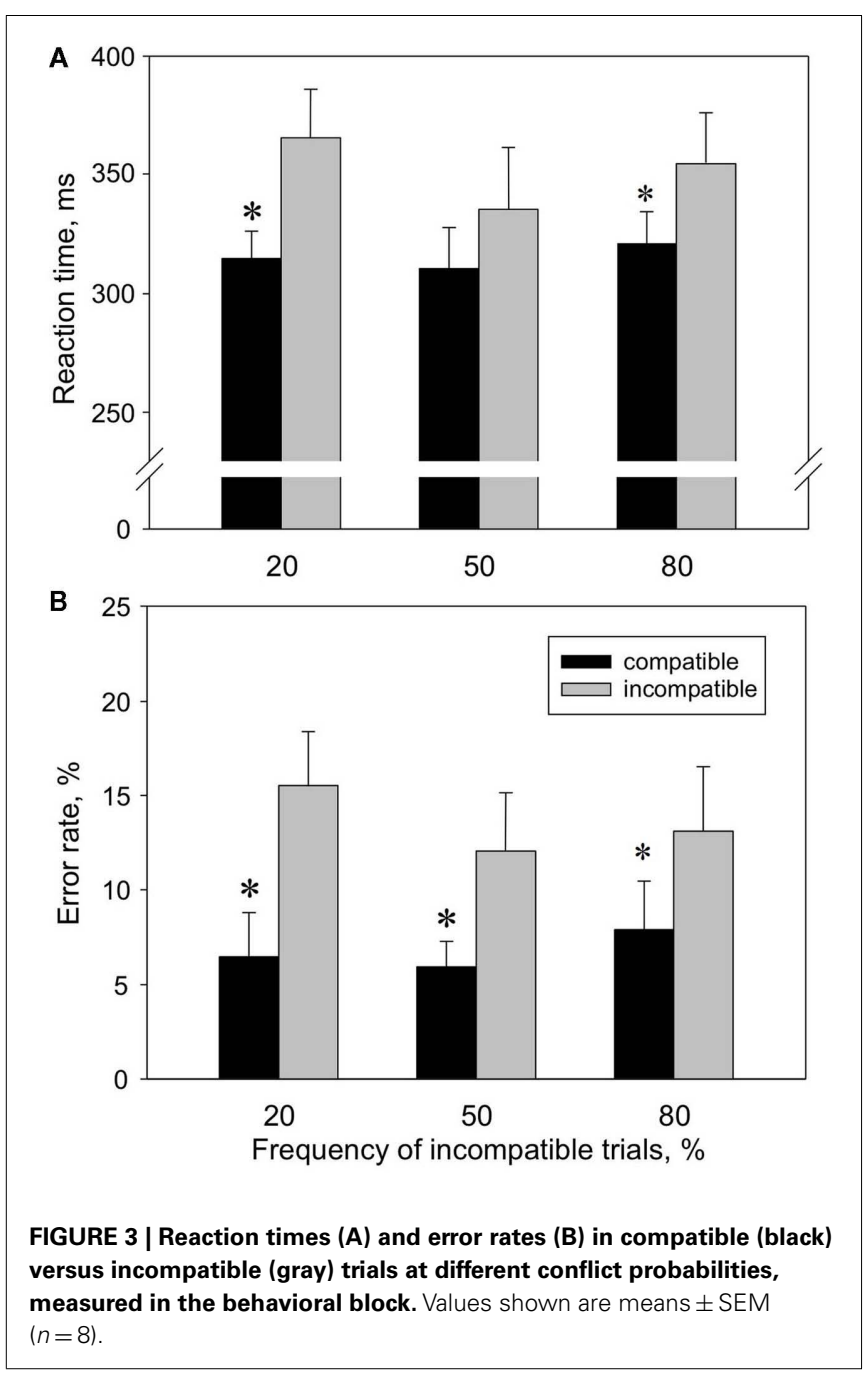

not differ significantly across tests $[F(4,12)=1.82, p=0.19$, oneway repeated measures ANOVA]. To further assess whether $T_{\mathrm{I}}$ is a valid control with high conflict level we compared average RTs during the first, middle, and last third (10 min each) of the task. During the first third, RTs were higher than in the other tasks, but decreased significantly during the following $20 \mathrm{~min}$ (Table 2). This indicates a high conflict level at least in the first 10 min of the task.

Error rates were on average 5.8\% lower in compatible compared to incompatible trials in $T_{\mathrm{R}}$, leading to a significant main effect across tests $[F(4,12)=4.58, p=0.0178]$ and a significant difference between compatible and incompatible trials of $T_{\mathrm{R}}$ after post hoc comparison.

Metabolic imaging. Subtractive approach. We first describe metabolic patterns associated with the Simon task in general, by comparing metabolic activity in one of the basic Simon task variants $\left(T_{\mathrm{I}}\right)$ with resting state $R_{\mathrm{S}}(n=7$; Figure 5$)$. We found a significant task related decrease of metabolic activity in the left prefrontal cortex (VOI statistics: one-sample $t$-test; $t=-3.33, p=0.0157$ ), right prelimbic cortex $(t=-2.74, p=0.0338)$, right posterior 

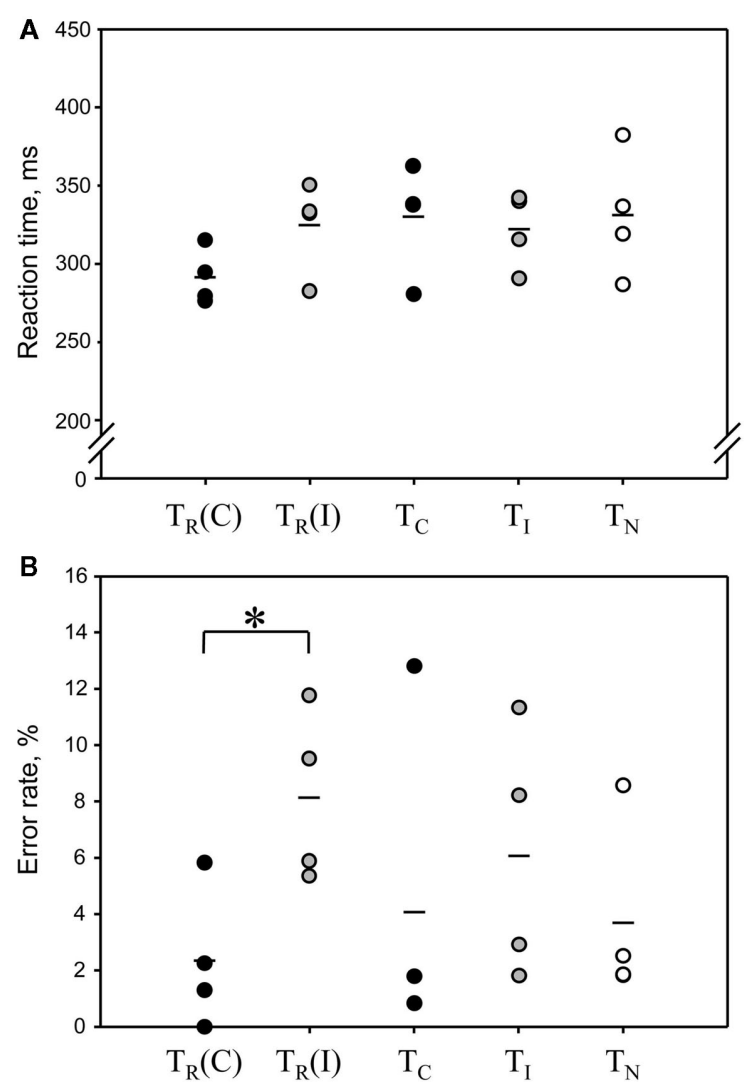

FIGURE 4 | Reaction times (A) and error rates (B) in the Simon tasks combined with PET imaging. Each dot represents the result from one animal $(n=4)$. Mean values are indicated by a short line. In the classical Simon task $T_{R}$, where compatible and incompatible trials were presented in randomized order, the results for compatible and incompatible trials were analyzed separately $\left[T_{R}(C)\right.$ and $\left.T_{R}(I)\right]$. There was a significant Simon effect on error rate, but not on reaction times. $T_{\mathrm{R}}(\mathrm{C})$ and $T_{\mathrm{R}}(\mathrm{I})$ correspond to black and gray bars, respectively, of the condition " $50 \%$ incompatible trials" in Figure 2. Control tasks: $T_{C}$, compatible trials only; $T_{1}$, incompatible trials only; $T_{N}$, neutral trials only.

Table 2 | Reaction times during PET sessions (mean \pm SEM).

\begin{tabular}{llll}
\hline Task & $\begin{array}{l}\text { First third of } \\
\text { trials }(\mathbf{m s})\end{array}$ & $\begin{array}{l}\text { Middle third of } \\
\text { trials }(\mathbf{m s})\end{array}$ & $\begin{array}{l}\text { Last third of } \\
\text { trials }(\mathbf{m s})\end{array}$ \\
\hline$T_{\mathrm{R}}$ & $326.2 \pm 16.9$ & $297.2 \pm 8.2$ & $303.2 \pm 15.9$ \\
$T_{\mathrm{C}}$ & $351.9 \pm 24.8$ & $323.1 \pm 19.6$ & $338.5 \pm 27.8$ \\
$T_{1}{ }^{*}$ & $361.9 \pm 24.7$ & $311.4 \pm 5.1$ & $299.1 \pm 15.7$ \\
$T_{N}$ & $342.7 \pm 18.5$ & $318.4 \pm 19.2$ & $335.1 \pm 36.1$ \\
\hline
\end{tabular}

${ }^{*} F(3,6)=5.46, p=0.045$ (one-way repeated measures ANOVA). One third comprises $10 \mathrm{~min}$.

cingulate region $\mathrm{Cg} 1(t=-3.66, p=0.0106)$ and decreased metabolic activity bilaterally in the posterior Cg2 (left: $t=-3.41$, $p=0.0144$; right: $t=-3.24, p=0.0176$ ). Furthermore, metabolic activity was decreased bilaterally in the retrosplenial granular cortex (left: $t=-2.65, p=0.0382$; right: $t=2.54, p=0.0442$ ), medial septum $(t=-4.22, p=0.0055)$, bilaterally in the lateral septum

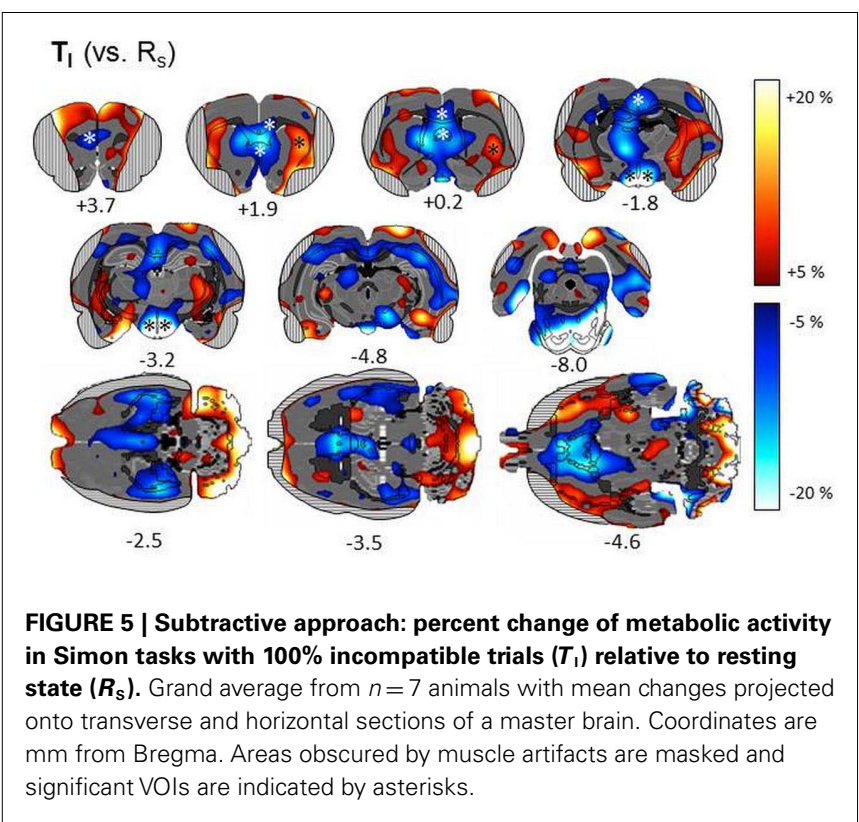

(left: $t=-4.71, p=0.0033$; right: $t=-3.86, p=0.0084$ ), and in the hypothalamus $(t<-3.0, p<0.022)$. A task related increase of metabolic activity was also found in the left lateral striatum $(t=3.22, p=0.0182)$.

Next, we report metabolic activity changes related to conflict processing. We looked for metabolic activity changes relative to $T_{\mathrm{N}}$, which were visible in $T_{\mathrm{I}}$ and $T_{\mathrm{R}}$, but not in $T_{\mathrm{C}}$ (Figure 6, columns 1-3; Table 1). In the right prelimbic cortex FDG uptake was significantly increased during $T_{\mathrm{R}}$ at the VOI level $(t=15.26$, $p=0.0006)$ as well as during $T_{\mathrm{I}}$ and $T_{\mathrm{R}}$ at voxel level $[\mathrm{F}(3,9)>4.3$, $p<0.0378$ ]. FDG uptake also increased significantly in the right ventrolateral striatum, on the border to the entorhinal cortex, during $T_{\mathrm{R}}[F(3,9)>4.98, p<0.0264$ for voxels $]$. In $T_{\mathrm{I}}$, there was a non-significant average increase of FDG uptake of more than $10 \%$ in the same region. Metabolic activity decreased in voxels of the left dorsocentral striatum during $T_{\mathrm{I}}$ and $T_{\mathrm{R}}[F(3,9)>4.5$, $p<0.0349$ ].

Brain activity changes related to potential automatic route facilitation caused by compatible trials should be visible in $T_{\mathrm{C}}$ and $T_{\mathrm{R}}$, but not in $T_{\mathrm{I}}$. We found decreased metabolic activity in right olfactory tubercle voxels during $T_{\mathrm{C}}$ and $T_{\mathrm{R}}[F(3,9)>4.6$, $p<0.0334]$.

The cerebrometabolic correlates of automatic route suppression may become most obvious if we analyze $T_{\mathrm{I}}$ relative to $T_{\mathrm{C}}$ (Figure 6, column 4). Here, we found increased FDG uptake in the right $\mathrm{Cg} 1$ region of the ACC, $(t>-3.39, p<0.0428$ for voxels), right orbitofrontal cortex $(t>-3.29 ; p<0.0462$ for voxels), right basal forebrain and nucleus accumbens $(t>-3.50$, $p<0.0393$ for voxels), right dorsolateral striatum $(t=-3.57$, $p=0.0374$ for one voxel; $t=13.4711, p=0.0006$ for VOI), right amygdala $(t>-3.35, p<0.0440$ for voxels), right subthalamic region ( $t>-4.11, p<0.0261$ for voxels), right mediodorsal thalamus ( $t>-3.37, p<0.0433$ for voxels), and left lateral hippocampus $(t>-5.22, p<0.0137$ for voxels). FDG uptake decreased in the left tenia tecta $(t>3.19, p<0.0497$ for voxels), right lateral 


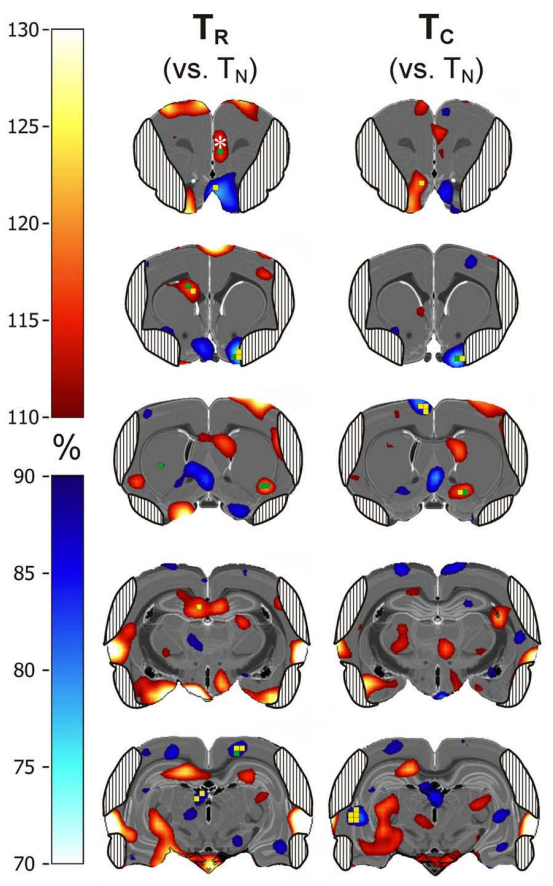

FIGURE 6 | Subtractive approach: percent change of metabolic activity in Simon tasks with randomized presentation of compatible and incompatible trials $\left(50 \%\right.$ each; $\left.T_{\mathrm{R}}\right), \mathbf{1 0 0} \%$ compatible trials $\left(T_{\mathrm{C}}\right)$, and $100 \%$ incompatible trials $\left(\boldsymbol{T}_{1}\right)$. Values are relative to $T_{\mathrm{N}}$ (column 1-3) or $T_{\mathrm{C}}$ (column 4). Grand average from $n=4$ animals with mean changes projected onto

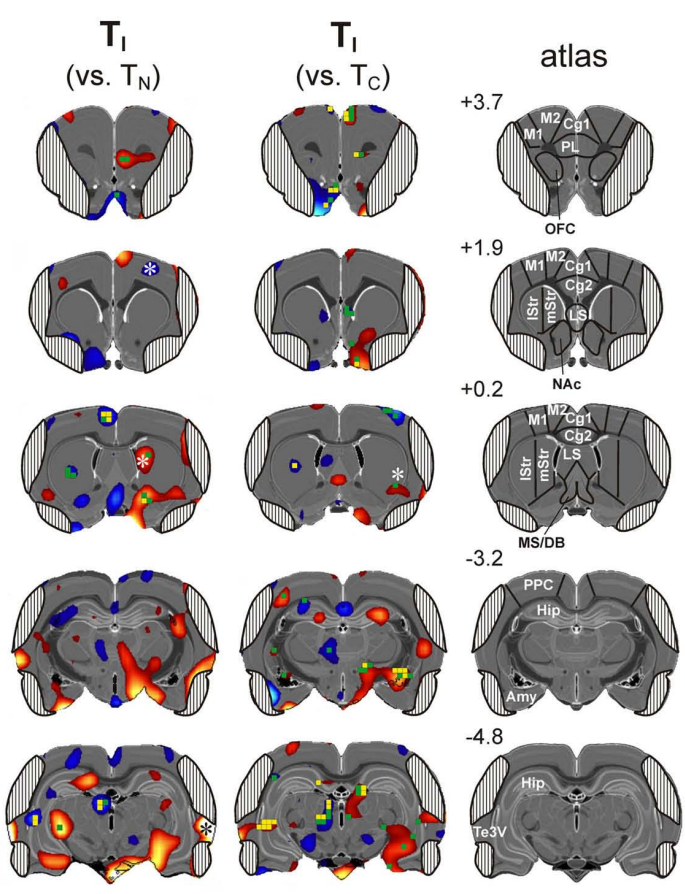

transverse sections of a master brain. Column 5: coordinates ( $\mathrm{mm}$ from Bregma) and analyzed volumes of interest (VOIs). Areas obscured by muscle artifacts are masked. Significant voxels (uncorrected $p<0.05$ ) are shown in green and (uncorrected $p<0.02$ ) in yellow, significant VOIs are indicated by asterisks. septum $(t>4.06, p<0.0270$ for voxels), left dorsocentral striatum $(t=5.45, p=0.0122$ for voxels $)$, left hippocampus $(t=3.75$, $p=0.0331$ for voxels), and left mediodorsal thalamus $(t>3.47$, $p<0.0403$ for voxels).

Correlative approach. Here the search was for areas where metabolic activity correlated with RT and/or ER during $T_{\mathrm{I}}$ and $T_{\mathrm{R}}$, but not during $T_{\mathrm{C}}$ and $T_{\mathrm{N}}$. The only area satisfying this precondition was the anterior premotor cortex aM2 (Figure 7), where metabolic activity was correlated to ER during $T_{\mathrm{I}}$ (left aM2: $R=0.99$, $p=0.0021$; right aM2: $R=0.99, p=0.0146)$ and inversely correlated to RT during $T_{\mathrm{R}}$ (left aM2: $R=-0.96, p=0.0440$; right aM2: $R=-0.86, p=0.1366$ ). This indicates that with a higher aM2 activity animals will respond faster during conflicting situations but at the cost of a higher error probability.

\section{DISCUSSION}

The purpose of the current study was twofold, namely (1) to identify and study brain regions involved in conflict processing in rodents, and (2) to demonstrate that behavioral FDG-PET is a suitable tool to analyze behavior on a metabolic level in a complex cognitive test like the Simon task.

\section{BEHAVIOR}

Our study confirmed the results of Courtière et al. (2007) that rats performing a Simon task displayed longer RTs and produced more errors in incompatible compared to compatible trials. This was statistically significant in the behavioral block, for both reaction times and ERs, while in the PET block only ERs were significantly elevated in incompatible trials. Taken together, behavioral results indicate that a cognitive conflict was present in incompatible trials, which was sufficient to produce metabolic changes in tasks with mixed compatible and incompatible trials in the PET situation. However, it is not possible to disentangle metabolic responses to compatible and incompatible trials within one PET session, because FDG-PET is a cumulative method. The uptake measured $1 \mathrm{~h}$ after FDG injection is heavily weighted to the total metabolic activity prevailing in the preceding interval when the animals were performing the task. We thus had to conduct control sessions in our PET study consisting of either $100 \%$ compatible $\left(T_{\mathrm{C}}\right)$ or $100 \%$ incompatible trials $\left(T_{\mathrm{I}}\right)$. In humans, the Simon effect is strongly reduced or even reversed in tasks that include $80 \%$ or more incompatible trials ("practice effect"; Stürmer et al., 2002; Melara et al., 2008; Iani et al., 2009), raising doubt whether the present experimental design generated a sufficient level of conflict in $T_{\mathrm{I}}$. However, a practice effect was not found in our rats, which displayed a significant Simon effect also in tasks with $80 \%$ incompatible trials, leading to the assumption that in $T_{\mathrm{I}}$ a high conflict level was present as well. It was therefore surprising that RTs during $T_{\mathrm{C}}$ and $T_{\mathrm{N}}$ were not reduced compared to $T_{\mathrm{I}}$. This may be explained by an order effect, since PET sessions were performed in the sequence $T_{\mathrm{N}}, T_{\mathrm{C}}, T_{\mathrm{I}}, T_{\mathrm{R}}$, on average 6 days apart with training in between. It may be possible that rats further improved their performance during the PET block, so that a 
A
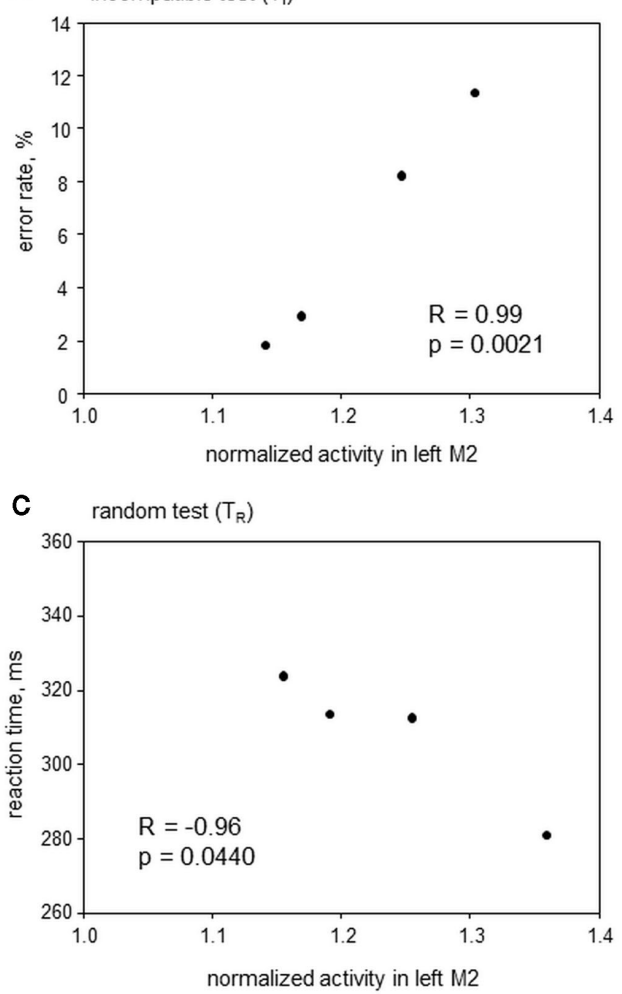

FIGURE 7 | Correlative approach: error rates and reaction times in Simon tasks containing incompatible trials $\left(T_{1}\right.$ and $\left.T_{\mathrm{R}}\right)$, plotted over metabolic
B incompatible test $\left(\mathrm{T}_{1}\right)$

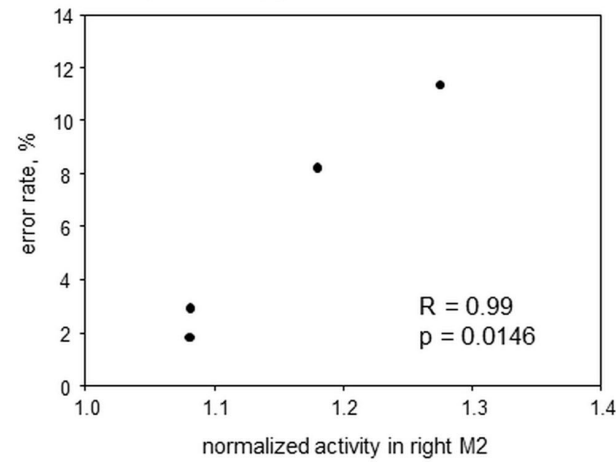

D

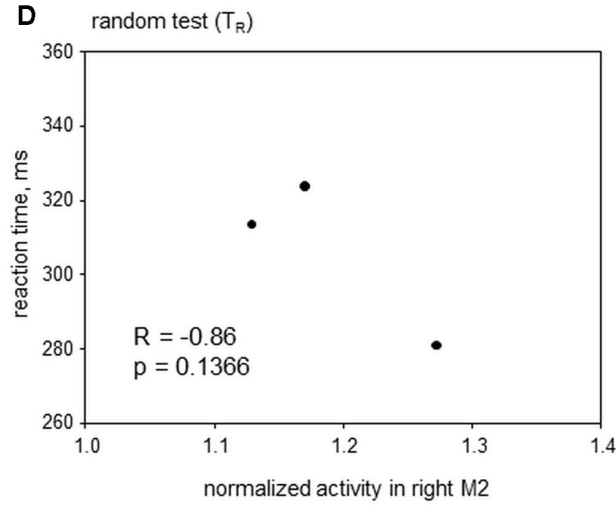

activity in the left $(A, C)$ and right $(B, D)$ anterior premotor cortex (aM2) of four animals. Significant correlations were found in (A-C). learning-induced decrease of RTs compensated a conflict-induced increase of RTs in $T_{\mathrm{I}}$.

\section{SIMON TASK VERSUS RESTING STATE}

According to numerous studies, energy consumption in a given brain area is mainly determined by input activity (for review see Ritter and Villringer, 2002; Raichle and Mintun, 2006) whereby excitatory and inhibitory inputs cannot be distinguished. Elevated FDG uptake can therefore be interpreted as increased afferent activity, independent of spike rate of the neurons in this area. In order to analyze brain activity changes during the Simon task in general, we compared FDG uptake during one of the Simon task variants with resting state $\left(R_{\mathrm{S}}\right)$. As Simon task we chose the task with $100 \%$ incompatible trials $\left(T_{\mathrm{I}}\right)$, because it was conducted by the most animals. We found a significant decrease of FDG uptake during the task in brain areas recently assigned to the default mode network (DMN) in rats (Lu et al., 2011), namely the prelimbic and cingulate cortex as well as the retrosplenial cortex. This corroborates human PET and fMRI studies demonstrating that the DMN contains areas with a high resting state activity, e.g., the posterior cingulate and anterior medial prefrontal cortices, which decrease their activity in attention-demanding cognitive tasks (Raichle et al., 2001; Greicius et al., 2003). In addition, FDG uptake decreased in the septum and the hypothalamus during $T_{\mathrm{I}}$ compared to $R_{S}$. This may reflect a stress-induced activation of these areas during $R_{\mathrm{S}}$ (Sung et al., 2009), because rats had not yet started training when the resting state scan took place and were therefore not as familiar with the operant chamber as during the Simon task scans.

\section{COMPARISON OF DIFFERENT SIMON TASK VARIANTS}

Brain areas involved in conflict processing are supposed to be activated when automatic and intentional route processing lead to different responses. These areas should therefore change their metabolic activity during PET sessions involving incompatible trials, i.e., during $T_{\mathrm{R}}$ and $T_{\mathrm{I}}$, but not during $T_{\mathrm{C}}$. As reference, a no-conflict control ( $T_{\mathrm{N}}$; bilateral stimulus presentation, therefore ambiguous spatial information) was used, and reported activity changes were relative to $T_{N}$, unless otherwise stated. We found a conflict related increase of FDG uptake in the prelimbic cortex. This is in line with other conflict studies on rats (de Wit et al., 2006; Haddon and Killcross, 2006), where the prelimbic cortex, together with the anterior part of the cingulate cortex, was essential for inhibiting the incorrect, competing response (Chudasama et al., 2003) and therefore seems to be involved in conflict resolution. FDG uptake also changed in the right ventrolateral striatum (increase) and the left dorsocentral striatum (decrease) during $T_{\mathrm{R}}$ and $T_{\mathrm{I}}$, indicating that these areas are related to conflict processing as well.

During the PET sessions involving compatible trials $\left(T_{\mathrm{R}}\right.$ and $T_{\mathrm{C}}$ ), possible automatic route facilitation may be reflected by increased FDG uptake. However, we found no spots of increased 
FDG uptake visible in $T_{\mathrm{R}}$ and $T_{\mathrm{C}}$ but not in $T_{\mathrm{I}}$. This indicates that facilitation, if present at all, was not strong enough to increase metabolic demand significantly. We therefore hoped to increase metabolic contrast between maximal conflict associated with automatic route suppression and minimal conflict possibly associated with automatic route facilitation by comparing $T_{\mathrm{I}}$ to $T_{\mathrm{C}}$ rather than to $T_{\mathrm{N}}$. A decrease of FDG uptake in $T_{\mathrm{I}}$ versus $T_{\mathrm{C}}$ could then be interpreted as decrease of excitatory activity of serially coupled automatic route areas. An increase of FDG uptake could reflect (1) an increase of inhibitory activity during automatic route suppression, or (2) an increase of excitatory activity reflecting conflict monitoring or resolution which was not strong enough to be visible in $T_{\mathrm{I}}$ or $T_{\mathrm{R}}$ versus $T_{\mathrm{N}}$. We found an increased FDG uptake in $T_{\mathrm{I}}$ relative to $T_{\mathrm{C}}$ in the right $\mathrm{Cg} 1$ region of the ACC, which was not visible in $T_{\mathrm{I}}$ relative to $T_{\mathrm{N}}$. This most likely indicates a conflict monitoring function in analogy to findings in human studies implicating this region as the main conflict monitoring area (Botvinick et al., 1999, 2004; Peterson et al., 2002; Kerns et al., 2004; Kerns, 2006). Furthermore, we found a significant decrease in FDG uptake in the right posterior motor cortex (pM1), suggesting that automatic route suppression may occur during the last stage of audiomotor integration. This is in line with an event-related potential study in humans, reporting "late" automatic route suppression in the frontolateral motor cortex (Stürmer and Leuthold, 2003). The increase of metabolic activity in the right dorsomedial striatum during $T_{\mathrm{I}}$ can as well be interpreted as evidence for conflict resolution via automatic route suppression, because this area is involved in behavioral inhibition (Eagle and Baunez, 2010). Furthermore, a small spot of decreased FDG uptake was observed during $T_{\mathrm{I}}$ (compared to both $T_{\mathrm{N}}$ and $T_{\mathrm{C}}$ ) and $T_{\mathrm{R}}$ in the dorsocentral striatum. This region receives projections from M2 and posterior parietal cortex (PPC; Cheatwood et al., 2005), and the fact that metabolic activity was also found to be lower in the ipsilateral M2 during $T_{\mathrm{I}}$, may therefore indicate blocking of the motor basal ganglia loop (Alexander and Crutcher, 1990; Joel and Weiner, 2000) during automatic route suppression.

So far we have not explained activity changes occurring in both $T_{\mathrm{I}}$ and $T_{\mathrm{C}}$ but not in the other tasks, such as the decrease of activity found in the left aM2 and right aM1. We preferred 100\% incompatible $\left(T_{\mathrm{I}}\right)$ or $100 \%$ compatible trials $\left(T_{\mathrm{C}}\right)$ over a mixed design, because metabolic patterns should be determined by one type of trials only. However, one could argue that in the homogeneous tasks $T_{\mathrm{C}}$ and $T_{\mathrm{I}}$ it could be possible for the animal to ignore the relevant stimulus dimension (pitch) and choose the correct response side with the help of the irrelevant stimulus dimension (side). In $T_{\mathrm{C}}$, the animal has to respond always toward the stimulus side, and in $T_{\mathrm{I}}$ always toward the opposite side. Because the rats have never encountered homogeneous tasks before, this new stimulus side - response side association, if established at all, must have been developed during the PET session itself. Especially in the purely incompatible $T_{\text {I }}$ task, we would then expect RTs to decrease continuously during the session, if the new rule based on stimulus side is easier for the animal than that based on pitch information. Indeed, we found a steady decrease of RTs during the $T_{\mathrm{I}}$ session. If the nature of audiomotor integration changes during the task, metabolic activity is supposed to be decreased in pathways related to the pitch-based intentional processing route compared to the neutral task $T_{\mathrm{N}}$, where animals have to rely solely on pitch information. In such a scenario, the decrease of activity found in the left aM2 and right aM1 may therefore be interpreted as linked to learning a new rule rather than caused by automatic route suppression. Although the correlative analysis (see below) supports the role of aM2 (but not aM1) in conflict processing, further studies should rather use a high conflict control with $10 \%$ compatible trials. The conflict level would still be high enough to determine metabolic patterns, but the rats will have to use the tone pitch response side association throughout the whole task. Interpreting metabolic patterns as associated with learning a new association would then be ruled out completely.

\section{CORRELATION OF IMAGING AND BEHAVIOR}

Conflict processing may not always be accompanied by profound changes of neuronal activity, which alter the average glucose metabolism by more than $5 \%$. Instead, activity changes may differ between animals, due to individual variations in conflict processing capacity, or may remain below our detection threshold. Thus, a correlative approach might provide further insights: Metabolic activity of a brain area involved in conflict processing may be correlated with behavioral parameters during $T_{\mathrm{I}}$ and/or $T_{\mathrm{R}}$, but not during $T_{\mathrm{C}}$ and $T_{\mathrm{N}}$. Consistent with this account, the activity of the left aM2 was inversely correlated to RTs during $T_{\mathrm{R}}$, indicating that fast animals had high premotor activities. Furthermore, these findings correspond well with data from humans suggesting that the premotor cortex is involved in conflict resolution (Egner et al., 2007).

\section{SYNOPSIS}

So far we have discussed activation patterns emerging in the Simon task versus resting state and comparisons between different variants of the Simon task independently from each other. But how can we interpret the finding that an area of the DMN, the prelimbic cortex, shows reduced metabolic activity during the Simon task with $100 \%$ conflict trials when compared to resting state, but increased activity when compared to the no-conflict Simon task control with $100 \%$ neutral trials (i.e., metabolic activity of prelimbic cortex: $\left.R_{\mathrm{S}}>T_{\mathrm{I}}>T_{\mathrm{N}}\right)$ ? Reduction of resting activity in the DMN during cognitive tasks is currently interpreted as attenuation of the brain's self-referential (excitatory) activity as a means of more effectively focusing on a task (Sheline et al., 2009). Our finding can therefore be explained in three different ways: (1) Metabolic activity changes comprise mainly alterations of excitatory input. DMN activity during $T_{\mathrm{N}}$ is therefore more strongly attenuated than during $T_{\mathrm{I}}$. (2) DMN activity is attenuated likewise during $T_{\mathrm{I}}$ and $T_{\mathrm{N}}$, but the prelimbic cortex is engaged in conflict processing during $T_{\mathrm{I}}$, leading to a higher net activity. (3) Metabolic activity changes reflect also inhibitory input activity. The prelimbic cortex may receive strong inhibitory input during $T_{\mathrm{I}}$, but not during $T_{\mathrm{N}}$, which would lead to an even more effective attenuation of resting activity during $T_{\mathrm{I}}$. Further studies are needed to decide which alternative may account for the observed activation patterns.

Our results demonstrate that spatial response conflicts occur in rats just as in humans. Our imaging results show remarkable similarities to the pattern of activated regions reported during 
conflict processing in human fMRI studies. The rat motor cortex (pM1) may be part of the automatic route or involved in its facilitation, while premotor (aM2), prelimbic, and ACC may play a role in conflict resolution and/or monitoring. Moreover, conflict-induced automatic route suppression presumably occurs

\section{REFERENCES}

Alexander, G. E., and Crutcher, M. D. (1990). Functional architecture of basal ganglia circuits: neural substrates of parallel processing. Trends Neurosci. 13, 266-271.

Arndt, S., Cizadlo, T., O’Leary, D., Gold, S., and Andreasen, N. C. (1996). Normalizing counts and cerebral blood flow intensity in functional imaging studies of the human brain. Neuroimage 3, 175-184.

Botvinick, M., Nystrom, L. E., Fissell, K., Carter, C. S., and Cohen, J. D. (1999). Conflict monitoring versus selection-for-action in anterior cingulate cortex. Nature 402, 179-181.

Botvinick, M. M., Braver, T. S., Barch, D. M., Carter, C. S., and Cohen, J. D. (2001). Conflict monitoring and cognitive control. Psychol. Rev. 108, 624-652.

Botvinick, M. M., Cohen, J. D., and Carter, C. S. (2004). Conflict monitoring and anterior cingulate cortex: an update. Trends Cogn. Sci. (Regul. Ed.) 8, 539-546.

Cheatwood, J. L., Corwin, J. V., and Reep, R. L. (2005). Overlap and interdigitation of cortical and thalamic afferents to dorsocentral striatum in the rat. Brain Res. 1036, 90-100.

Chudasama, Y., Passetti, F., Rhodes, S. E. V., Lopian, D., Desai, A., and Robbins, T. W. (2003). Dissociable aspects of performance on the 5-choice serial reaction time task following lesions of the dorsal anterior cingulate, infralimbic and orbitofrontal cortex in the rat: differential effects on selectivity, impulsivity and compulsivity. Behav. Brain Res. 146, 105-119.

Courtière, A., Hardouin, J., Burle, B., Vidal, F., and Hasbroucq, T. (2007). Simon effect in the rat: a new model for studying the neural bases of the dual-route architecture. Behav. Brain Res. 179, 69-75.

De Jong, R., Liang, C. C., and Lauber, E. (1994). Conditional and unconditional automaticity: a dual-process model of effects of spatial stimulusresponse correspondence. J. Exp. Psychol. Hum. Percept. Perform. 20, 731-750.

de Wit, S., Kosaki, Y., Balleine, B. W., and Dickinson, A. (2006). Dorsomedial prefrontal cortex resolves response conflict in rats. J. Neurosci. 26, 5224-5229.
Eagle, D. M., and Baunez, C. (2010). Is there an inhibitory-responsecontrol system in the rat? Evidence from anatomical and pharmacological studies of behavioral inhibition. Neurosci. Biobehav. Rev. 34, 50-72.

Egner, T., Delano, M., and Hirsch, J. (2007). Separate conflict-specific cognitive control mechanisms in the human brain. Neuroimage 35, 940-948.

Endepols, H., Sommer, S., Backes, H., Wiedermann, D., Graf, R., and Hauber, W. (2010). Effort-based decision making in the rat: an [18F]fluorodeoxyglucose micro positron emission tomography study. J. Neurosci. 30, 9708-9714.

Forstmann, B. U., van den Wildenberg, W. P., and Ridderinkhof, K. R. (2008). Neural mechanisms, temporal dynamics, and individual differences in interference control. J. Cogn. Neurosci. 20, 1854-1865.

Genovese, C. R., Lazar, N. A., and Nichols, T. (2002). Thresholding of statistical maps in functional neuroimaging using the false discovery rate. Neuroimage 15, 870-878.

Greicius, M. D., Krasnow, B., Reiss, A. L., and Menon, V. (2003). Functional connectivity in the resting brain: a network analysis of the default mode hypothesis. Proc. Natl. Acad. Sci. U.S.A. 100, 253-258.

Haddon, J. E., and Killcross, S. (2006). Prefrontal cortex lesions disrupt the contextual control of response conflict. J. Neurosci. 26, 2933-2940.

Hommel, B., Proctor, R. W., and Vu, K. P. (2004). A feature-integration account of sequential effects in the Simon task. Psychol. Res. 68, 1-17.

Iani, C., Rubichi, S., Gherri, E., and Nicoletti, R. (2009). Co-occurrence of sequential and practice effects in the Simon task: evidence for two independent mechanisms affecting response selection. Mem. Cognit. 37, 358-367.

Jang, D. P., Lee, S. H., Lee, S. Y., Park, C. W., Cho, Z. H., and Kim, Y. B. (2009). Neural responses of rats in the forced swimming test: A [F-18]FDG micro PET study. Behav. Brain Res. 203, 43-47.

Joel, D., and Weiner, I. (2000). The connections of the dopaminergic system with the striatum in rats and primates: an analysis with respect to the functional and compartmental

in M2 and M1 as well as in the dorsocentral striatum, i.e., on the motor side of audiomotor integration. Our data shows interesting insights in cross species comparison of conflict processing, and opens novel opportunities to investigate the anatomical basis of conflict processing in a rodent model.

organization of the striatum. Neuroscience $96,451-474$.

Kerns, J. G. (2006). Anterior cingulate and prefrontal cortex activity in an FMRI study of trial-to-trial adjustments on the Simon task. Neuroimage 33, 399-405.

Kerns, J. G., Cohen, J. D., MacDonald, A. W., Cho, R. Y., Stenger, V. A., and Carter, C. S. (2004). Anterior cingulate conflict monitoring and adjustments in control. Science 303 1023-1026.

Lu, H., Zou, Q., Rea, W., Stein, E. A., and Yang, Y. (2011). Rat brain possesses a default mode network. Proc. Int. Soc. Magn. Reson. Med. 19, 3684.

Melara, R. D., Wang, H. J., Vu, K. P. L. and Proctor, R. W. (2008). Attentional origins of the Simon effect: behavioral and electrophysiological evidence. Brain. Res. 1215, 147-159.

Nichols, T., and Hayasaka, S. (2003). Controlling the familywise error rate in functional neuroimaging: a comparative review. Stat. Methods Med. Res. 12, 419-446.

Paxinos, G., and Watson, C. (2005). The Rat Brain in Stereotaxic Coordinates: The New Coronal Set, 5th Edn. San Diego: Elsevier Academic Press, 209.

Peterson, B. S., Kane, M. J., Alexander, G. M., Lacadie, C., Skudlarski, P., Leung, H. C., May, J., and Gore, J. C. (2002). An event-related functional MRI study comparing interference effects in the Simon and Stroop tasks. Brain Res. Cogn. Brain Res. 13, 427-440.

Qi, J., Leahy, R. M., Cherry, S. R. Chatziioannou, A., and Farquhar T. H. (1998). High-resolution 3D Bayesian image reconstruction using the microPET small-animal scanner. Phys. Med. Biol. 43, 1001-1013.

Raichle, M. E., MacLeod, A. M., Snyder, A. Z., Powers, W. J., Gusnard, D. A., and Shulman, G. L. (2001). A default mode of brain function. Proc. Natl. Acad. Sci. U.S.A. 98, 676-682.

Raichle, M. E., and Mintun, M. A. (2006). Brain work and brain imaging. Annu. Rev. Neurosci. 29, 449-476.

Ritter, P., and Villringer, A. (2002). Inhibition and functional magnetic resonance imaging. Int. Congr. 1235, 213-222.

Robbins, T. W., Muir, J. L., Killcross, A. S., and Pretsell, D. (1993). "Methods for assessing attention and stimulus control in the rat," in Behavioural Neuroscience: A Practical Approach, ed. A. Sahgal (Oxford: IRL Press).

Rocke, D. M., Goldberg, Z., Schweitert, C., and Santana,A. (2005). A method for detection of differential gene expression in the presence of interindividual variability in response. Bioinformatics 21, 3990-3992.

Sheline, Y. I., Barch, D. M., Price, J. L. Rundle, M. M., Vaishnavi, S. N., Snyder, A. Z., Mintun, M. A., Wang, S., Coalson, R. S., and Raichle, M. E. (2009). The default mode network and self-referential processes in depression. Proc. Natl. Acad. Sci. U.S.A. 106, 1942-1947.

Simon, J. R., Hinrichs, J. V., and Craft, J. L. (1970). Auditory S-R compatibility: reaction time as a function of ear-hand correspondence, and ear-response location correspondence. J. Exp. Psychol. 86, 197-202.

Stürmer, B., and Leuthold, H. (2003). Control over response priming in visuomotor processing: a lateralized event-related potential study. Exp. Brain Res. 153, 35-44.

Stürmer, B., Leuthold, H., Soetens, E., Schroter, H., and Sommer, W. (2002). Control over location-based response activation in the Simon task: behavioral and electrophysiological evidence. J. Exp. Psychol. Hum. Percept. Perform. 28, 1345-1363.

Sung, K. K., Jang, D. P., Lee, S., Kim, M., Lee, S. Y., Kim, Y. B., Park, C. W., and Cho, Z. H. (2009). Neural responses in rat brain during acute immobilization stress: a F-18 FDG micro PET imaging study. Neuroimage 44, 1074-1080.

Swanson, L. (2003). Brain Maps: Structure of the Rat Brain. 3rd edn. San Diego: Academic Press.

Vollmar, S., Hampl, J. A., Kracht, L., and Herholz, K. (2007). "Integration of functional data (PET) into brain surgery planning and neuronavigation," in Advances in Medical Engineering, eds T. M. Buzug, D. Holz, J. Bongartz, M. KohlBareis, and U. Hartmann (Berlin: SpringerVerlag), 98-103.

Wienhard, K. (2002). Measurement of glucose consumption using [18F]fluorodeoxyglucose. Methods 27, 218-225. 
Zhang, H. H., Zhang, J., and Kornblum, S. (1999). A parallel distributed processing model of stimulusstimulus and stimulus-response compatibility. Cogn. Psychol. 38, 386-432.

Conflict of Interest Statement: The authors declare that the research was conducted in the absence of any commercial or financial relationships that could be construed as a potential conflict of interest.

Received: 12 October 2011; accepted: 23 January 2012; published online: 09 February 2012
Citation: Marx C, Lex B, Calaminus $C$, Hauber $W$, Backes $H$, Neumaier B, Mies G, Graf $R$ and Endepols $H$ (2012) Conflict processing in the rat brain: behavioral analysis and functional $\mu P E T$ imaging using $\left[{ }^{18}\right.$ F]fluorodeoxyglucose. Front. Behav. Neurosci. 6:4. doi: 10.3389/fnbeh.2012. 00004
Copyright (c) 2012 Marx, Lex, Calaminus, Hauber, Backes, Neumaier, Mies, Graf and Endepols. This is an open-access article distributed under the terms of the Creative Commons Attribution Non Commercial License, which permits noncommercial use, distribution, and reproduction in other forums, provided the original authors and source are credited. 